DE DE GRUYTER

OPEN

G

BULGARIAN ACADEMY OF SCIENCES

CYBERNETICS AND INFORMATION TECHNOLOGIES • Volume 16, No 6

Special issue with selection of extended papers from 6th International Conference on Logistic, Informatics and Service Science LISS'2016

Sofia • 2016

Print ISSN: 1311-9702; Online ISSN: 1314-4081

DOI: $10.1515 /$ cait-2016-0093

\title{
Realization of Humanoid Robot Playing Golf
}

\author{
Xibao Wu, Wenbai Chen, Weichen Li \\ Beijing Information Science \& Technology University, 100192, China \\ E-mail: wuxibao@bistu.edu.cn chenwb@bistu.edu.cn
}

\begin{abstract}
Aiming at the golf tournament technical requirements in International Humanoid robot Olympic Game (IHOG), two-freedom-degrees "head \& eye" system based on monocular vision robot MF-AI is developed. The new robot equipped with the "head \& eye" system can identify the location of the golf ball and the hole, and can measure the distance of the two objects. Experiments show that the two-freedom-degrees "head \& eye" system improves the accuracy of hitting the golf ball into the hole. Moreover, the system can identify the target, can calibrate distances, can measure robot's moving and look for the hole, can adjust the position of the robot and hit the ball effectively.
\end{abstract}

Keywords: Humanoid robot, monocular vision, international humanoid robot, olympic game.

\section{Introduction}

Humanoid robots have been greatly developed in recent years. A robot with ability for dancing in response to music could lead to novel and interesting interactions with humans [1-3]. For example, in 2007 Toyota unveiled that robots could play the trumpet and violin in orchestras [2]. In 2008, the Honda robot ASIMO conducted the Detroit Symphony Orchestra, and in 2009, robot-actors were used in a theater production in Osaka, Japan [3-5].

International Humanoid robot Olympic Game (IHOG) is another kind of international competitive entertainment activities by using small humanoid robots to be athletes with the aid of human Olympic competition rules. The aim of IHOG is promoting the key technology research and development of humanoid robots with the help of human Olympic charm and challenges, and to realize robot era eventually $[6,7]$.

There are 24 kinds of competitions in 6 categories like sports, entertainment, art and construction. The key technology of these competition events contains the basics of technology of future humanoid type service robot [7]. Sport games in 
IHOG include sprint, long distance, fencing and others. Golf is one of the hardest games. Golf has a high demand of requirements to the ball hitting strength and angle; the robot needs to calculate angle, strength and distance to the target accurately, in order to complete this action.

According to the golf tournament technical requirements in International Humanoid robot Olympic Game, this paper developed two-freedom-degrees "head \& eye" system which is based on MF-AI robot with monocular vision. The MF-AI robot only identifies the target in front of it. The new system can identify the location of the golf ball and the hole, and can measure the distance of two locations. Experiments show that the two-freedom-degrees "head \& eye" system can complete the goal, can calibrate distances, can measure robot's moving, and robot's looking for the hole, robot positioning processes are fine-tuned. The system can improve the accuracy of hitting the golf ball into the hole effectively.

\section{Technical requirements of golf game in IHOG}

\subsection{The rules of the game}

- The competition item mainly tests the planning ability of robots to the complex actions of the golf sport (beating in ball) and vision-based intelligent control technology.

- The difference of the competition from basketball and football is that special ball bars are used to hit the ball into a hole as in golf sport.

- The competition process is: firstly, the robot uses vision to see a ball and a hole, and to determine the mutual distance and position, then, the two arms are cooperated to hit a ball into the hole; if the ball is hit into the hole, the robot obtains score, otherwise, the robot does not obtain score, and the robot with the biggest score is the winner.

- In the game process, if the robot falls, or cannot grasp the ball bar, or cannot hit the ball, the robot is considered as "failed" and cannot obtain score.

- Competition field size is shown on Fig. 1.

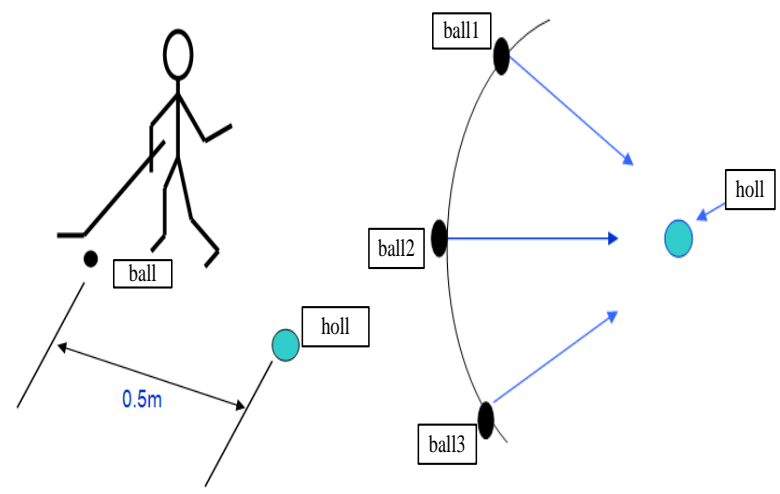

Fig. 1. Humanoid robot playing golf 


\subsection{Single visual system}

The visual system described here uses the research of single visual system of humanoid robot. Imaging principle of the ranging system is mainly used for small hole imaging.

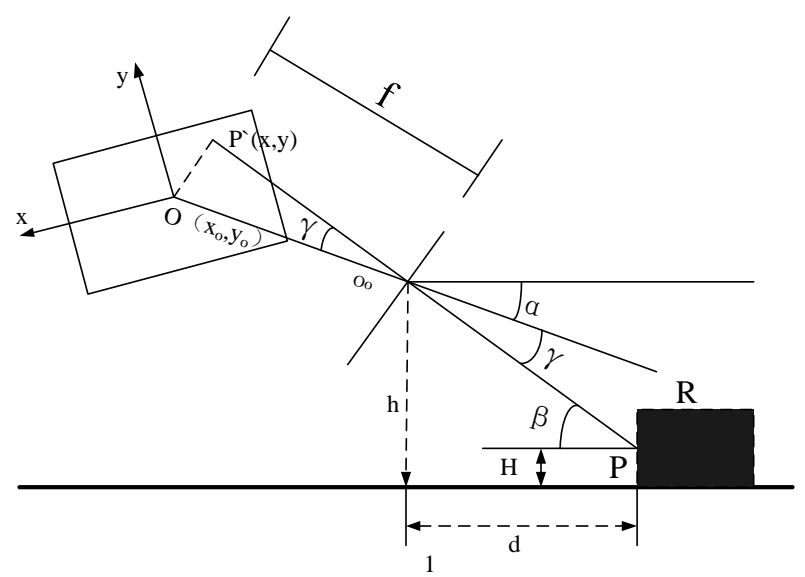

Fig. 2. Principle of small hole imaging

As shown in Fig. 2, $R$ is the golf ball, effective focal length is marked as $f$ of the CCD camera mounted on top of the robot, pitch Angle is $\alpha$, the height of the ground is $h$; the measuring point is $P$, the horizontal distance of $P$ and the centre of the lens is $d$.

Among them, $O_{0}$ is the lens centre, $O\left(x_{0}, y_{0}\right)$ is the intersection of the optical axis and the image plane, $P(x, y)$ is the projection of $P$ in the image plane.

The geometric relationships,

$$
\begin{gathered}
\beta=\alpha+\gamma, \\
\operatorname{tg} \beta=(h-H) / d, \\
\operatorname{tg} \gamma=O P^{\prime} / f .
\end{gathered}
$$

Simultaneous (1), (2) and (3), thus

$$
d=(h-H) / \operatorname{tg}(\alpha+\gamma)=(h-H) / \operatorname{tg}\left[\alpha+\operatorname{arctg}\left(O P^{`} / f\right)\right],
$$

where

$$
O P^{\prime 2}=y^{2}+x^{2},
$$

$d x$ and $d y$ are set as the physical size of a pixel corresponding to the image plane on the $x$-axis and $y$-axis; thus

$$
\left[\begin{array}{l}
u \\
v \\
1
\end{array}\right]=\left[\begin{array}{ccc}
1 / d & 0 & u_{0} \\
0 & 1 / d & v_{0} \\
0 & 0 & 1
\end{array}\right]\left[\begin{array}{l}
x \\
y \\
1
\end{array}\right],
$$

then 


$$
x=\left(u-u_{0}\right) d_{x}, y=\left(v-v_{0}\right) d_{y} .
$$

In (5),

$$
O P^{2}=\left[\left(u-u_{0}\right) d_{x}\right]^{2}+\left[\left(v-v_{0}\right) d_{y}\right]^{2}
$$

Assuming that

$$
f_{x}=f / d_{x}, f_{y}=f / d_{y},\left(O P^{\prime} / f\right)^{2}=\left[\left(u-u_{0}\right) / f_{x}\right]^{2}+\left[\left(v-v_{0}\right) / f_{y}\right]^{2},
$$

simultaneously to (4) and (9), we can obtain the distance between the point $P$ and the camera

$$
d=(h-H) / \operatorname{tg}\left[a+\operatorname{arctg} \sqrt{\left[\left(u-u_{0}\right) / f_{x}\right]^{2}+\left[\left(v-v_{0}\right) / f_{y}\right]^{2}}\right] .
$$

\section{Structure of humanoid robot}

As shown on Fig. 3, metal fighter is a new biped humanoid robot, which uses Atmega128 to control the joint rotation with 16 digital servo motors. The size of Metal Fighter is $325 \times 200 \times 100 \mathrm{~mm}$, its weight is $1.2 \mathrm{KG}$, and its digital servo motor has $9 \mathrm{KG}$ torque. In addition, the metal fighter's feet not only can walk, but also can exhibit a variety of difficult humanoid movements, such as running, roll, pre-roll, and so on. So, it is suitable for robot competition, teaching and entertainment.
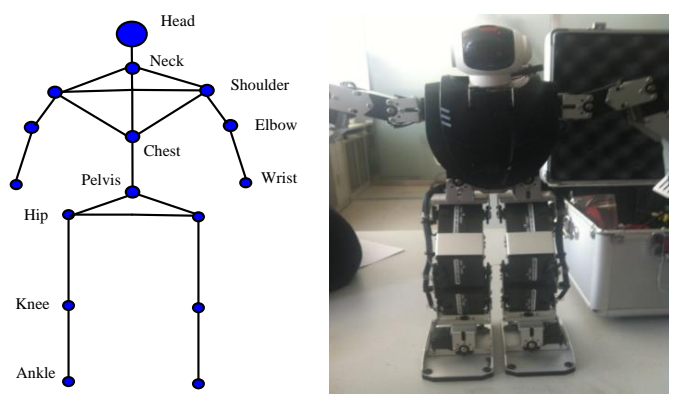

Fig. 3. Frame of metal fighter

Freedom degree is the number of independent variables which can complete describe the mechanical motion of the system. For the Metal Fighter, the number of free joints is illustrated in Table 1.

Table 1. Freedom setting of metal fighter

\begin{tabular}{|c|c|c|}
\hline \multirow{2}{*}{ Arm } & Shoulder & $2-\mathrm{DOF}$ \\
\cline { 2 - 3 } & Elbow & 1-DOF \\
\hline \multirow{3}{*}{ Leg } & Hip & 2-DOF \\
\cline { 2 - 3 } & Knee & 1-DOF \\
\cline { 2 - 3 } & Ankle & 2 -DOF \\
\hline Total & \multicolumn{2}{|c|}{$8 \times 2=16$ DOF } \\
\hline
\end{tabular}

Because of the limitation of physical condition, the space of the robot including its every part cannot exceed the mechanical structure range. The key parameter of robot behavior range is illustrated in Table 2 . 
Table 2. Key parameter of the metal fighter

\begin{tabular}{|c|c|c|c|}
\hline Joint & $\begin{array}{c}\text { Range of motion } \\
\text { (angle magnitude) }\end{array}$ & Joint & $\begin{array}{c}\text { Range of motion } \\
\text { (angle magnitude) }\end{array}$ \\
\hline Lateral shoulder & $-20-160$ & Forward shoulder & $-40-140$ \\
\hline Elbow & $0-90$ & Forward hip & $-90-80$ \\
\hline Lateral hip & $-30-90$ & Knee & $-90-90$ \\
\hline Forward ankle & $-90-90$ & Lateral ankle & $-30-90$ \\
\hline
\end{tabular}

\section{Gait planning of humanoid robot}

Motion planning of biped robot mainly includes the simple act planning and complicated motion planning. Simple act planning includes lifting the arms, squatting down, standing up, turning etc., and complicated motion planning includes weight lifting, sprint, golf, etc. Through the analysis of the basic act planning, we can research the complex movement further.

\subsection{Walking motion planning}

The most basic action of the biped robots on foot is to walk forward. In slow motion planning, the biped robots walk through two main phases: single leg support and both legs support. It is easy to control the robots' centre of gravity when the robots use both legs to stand, but the feasibility adjustment of the centre of gravity from both legs standing to single leg support is a difficult problem in the process of robots' advancement. In order to implement the smooth transition from both legs support to single leg support, the robots' position and attitude have to be adjusted to incline towards the area of single leg support from the centre stage of both legs support before motion planning of biped robot.

As a result, the slow walk of the biped robots only contains starting gait, normal gait, and stopping the gait. In the process of movement, each joints of bipedal robots maintain static equilibrium conditions, namely, bipedal robots' centre of gravity always stays in the stable area of the support leg, and doesn't consider the effect of inertia force. It is supposed that the robots' walk way is simple walk. The bipedal robots begin in the standard standing posture. The body moves towards the right to make the robot centre of gravity to right foot support area, and then, the left leg steps out through adjusting the leg joint servo motor. The centre of gravity in the support area of right leg is kept constant, and the left foot falls to the ground at the same time the centre of gravity moves towards left front to reach the support area of left foot. Then, the right leg lifts to maintain the centre of gravity in the support area of left foot, and the right foot falls to the ground at the same time the centre of gravity moves towards right front to reach the support area of right foot. Finally, the robot takes back its left foot and restores standard standing postures. A complete walk process ends and the process of the biped robots' walk step by step is shown as follows. 

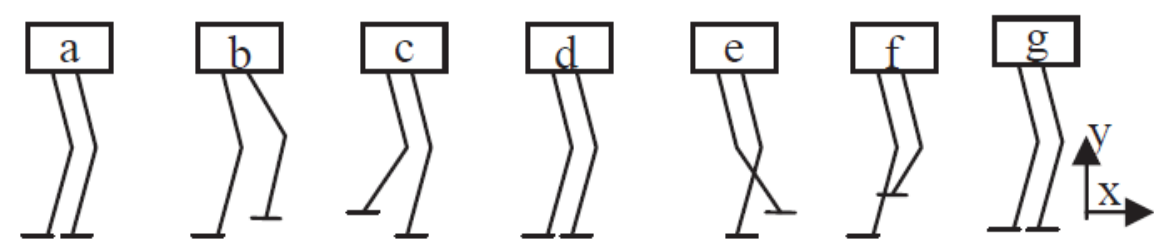

Fig. 4. Biped robot walking step by step

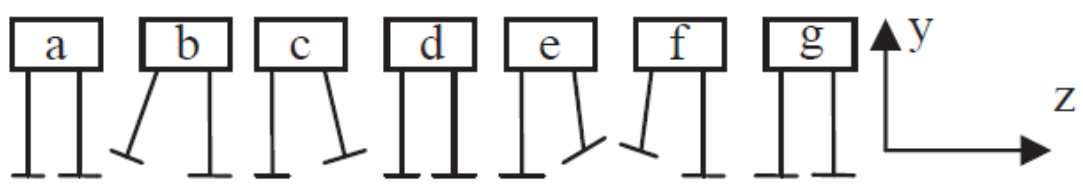

Fig. 5. Biped robot Lateral walking step by step

Lateral walk as shown on Fig. 5 is similar to walk forward. Fig. 5 shows the biped robot's standard standing posture at the beginning of walk. b-sketch presents that the centre of gravity moves towards the left foot and the left foot takes a step. c-sketch shows that the left foot falls to the ground and at the same time the centre of gravity moves towards left front to reach the support area of both legs. d-sketch expresses that the centre of gravity is in the support area of left leg. e-sketch is the situation that the left foot takes a step. f-sketch shows that the right foot falls to the ground at the same time the centre of gravity reaches the support area of both shoulders. g-sketch is the standard standing posture after a walk process is completed.

The key of walk is gait planning based on the bipedal robot's centre of gravity, so the design method employs the way of substep based on the centre of gravity. The way resolves walking process into several independent actions, and adjusts the location of the centre of gravity of robots' various actions to complete the bipedal robots' slow motion planning.

\subsection{Turning action planning}

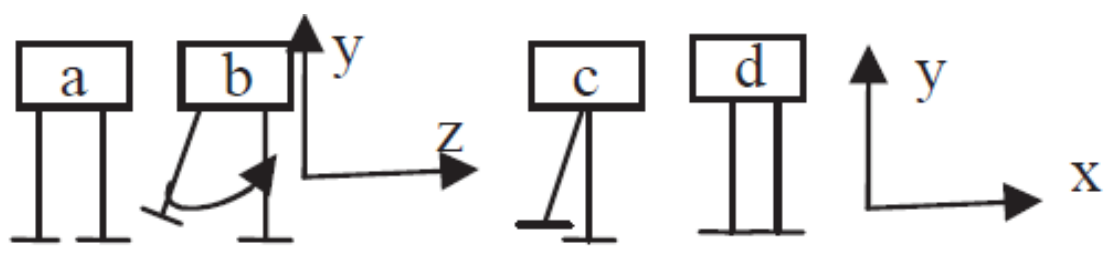

Fig. 6. Biped robot turning step by step

Bipedal robots' turning action is shown on Fig. 6. First, when the bipedal robot turns to left, its body inclines towards left; at the same time the centre of gravity moves towards left to reach the support area of left leg. Then, the bipedal robot steps to its right foot forward, and controls servo motor to make the right foot fall to the ground. Finally, the motor servo of right leg recovers, and the bipedal robot implements the action of turning left through the friction between the robot's foot motherboard and the ground. 


\subsection{Lifting arm action planning}

Lifting arm action planning could influence rotation control of robot's both arms joint servo motors. The bipedal robot's centre of gravity will rise when both arms joint servo motors rotate, to make both arms lift. However, the bipedal robot's center of gravity is in the support area of both legs, and legs joint servo motors are not controlled to rotate. Therefore, the bipedal robot needs a signal of servo motor to rotate legs joint servo motors, in order to keep the stable stand.

\section{The design scheme of humanoid robot golf game system}

Humanoid robot golf game system can be divided into three parts according to the hardware part, intelligent robot, PC and communication module. Robot Basic is an underlying program that can write all kinds of robot motions. It can achieve a series of actions which the robot needs to complete in the golf game, like finding the moving ball, attitude, adjustment, swing, hitting.

PC completes image processing, in particular smoothing the image, color recognition and feature extraction. PC also plans robot motion according to the needs of golf game, and finally realizes the complete set of movements of the golf project. The communication module mainly completes the image and instructions transmission. The five major Close-transmission technologies are $2.4 \mathrm{G}$ wireless digital chip, Zig Bee, Wi-Fi, Bluetooth, IrDA; and the new system uses $2.4 \mathrm{G}$ digital chip for the communication mode of images, and Bluetooth for the communication mode of robot commands. The overall structure is shown on Fig. 7.

\subsection{Hardware design}

The new system is designed on MF-AI3 monocular visual prototype robot. Monocular visual MF-AI robots, as shown on Fig. 4 have only Single freedom "head-eye" system. Because of the robot's target recognition only with target ahead, it can't execute higher requirements of the visual tasks needed for golf game; the robot cannot identify the golf ball and the hole at the same time. We need a twofreedom-degrees "head \& eye" system to let the robot find a ball and a hole. This is the only way to let a ball be hilted into a hole.

Considering the robot hardware compatibility issues, the new system uses a complete set of steering form prototype robot, MRS-D2009SP, MRS-D1812SP respectively. There is no big difference in performance between the two kinds of steering gear, but there are certain differences in overall dimensions. MRS-D1812SP and MRS-D2009SP use the same size of steering wheel which has $18 \mathrm{~mm}$ outside diameter and $8 \mathrm{~mm}$ inside diameter. But MRS-D1812SP and MRSD2009SP have a big gap between pitman arm spacing. MRS-D1812SP's pitman arm spacing is only $30 \mathrm{~mm}$ while MRS-D2009SP pitman arm spacing is $50 \mathrm{~mm}$. Using the above data, the corresponding fitting material object is made as shown on Fig. 8. 


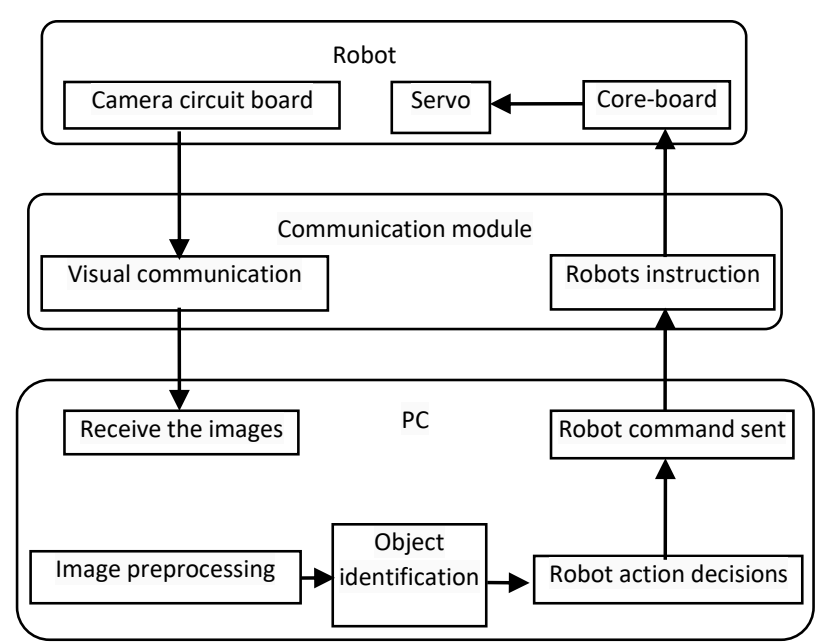

Fig. 7. Overall system structure

MRS-D2009SP steering gear installed in the robot body is responsible for the head movement along the $X$ axis, and controls the head turn around left and right. Then MRS-D1812SP steering gear is installed upper, and is responsible for the head movement along the $Y$ axis; it controls the head moving up and down. Fittings fixed on the MRS-D1812SP steering gear are fixed on the head of the robot in that way. The overall physical diagram of the two-freedom-degrees "head \& eye" system is shown on Fig. 9.

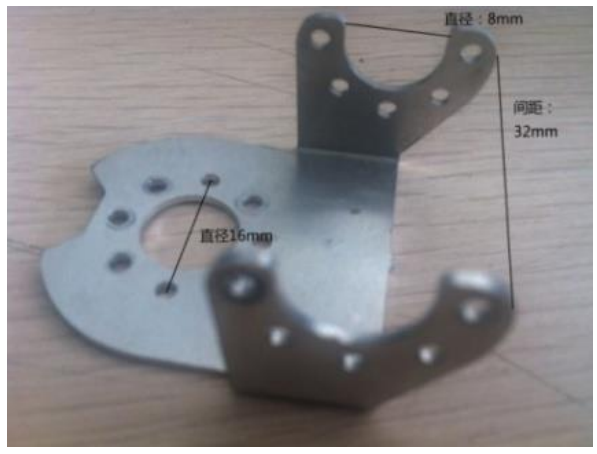

Fig. 8. Physical figure of steering gear

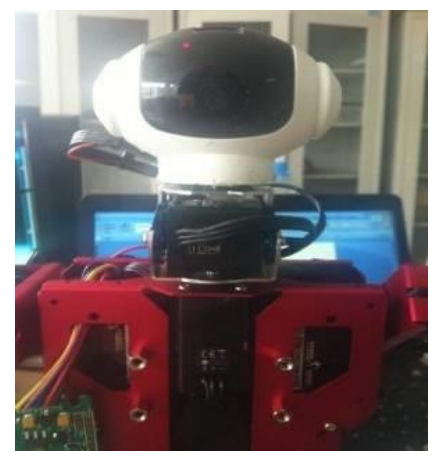

Fig. 9. Physical diagram

\subsection{Software design}

Software design includes two parts, bottom instructions received and upper PC image transmission and processing.

\subsubsection{Robot side}

On the base of received instructions, the robot perform motor control due to RobotBasic software programming. RobotBasic development environment can realize robot program compiling, debugging and download. Also, capture and real- 
time control can be stated on each joint of the robot motor. RobotBasic language has been added to the robot basic programming language [8].

The robot is bobbing its head owing to the two-freedom-degrees "head \& eye" system under the control of the power steering gear, and under steering gear the robot is able to turn right or left.

\subsubsection{PC side}

The robot motion is decided by upper PC software implementation controlling the intelligent robot; Upper PC uses $\mathrm{C}^{++}$language. Upper PC mainly completes the three tasks: to process the image acquisition and recognition of color [9-11] through the OpenCV for the robot; to connect the robot control system and write robot control instructions; to write MFC program allowing the user to control the robot with the mouse.

Image acquisition and processing is the key to complete the task. They are divided into the following four parts:

(1) Initialization, including reading image, image storage, etc.

(2) Pre-treatment, including color space transformation, image smoothing, etc.

(3) Extraction of environment features and target recognition.

(4) Drawing image to facilitate the later strategy.

\section{The system test}

Hitting the golf ball is an example to test the system. The golf ball hitting process includes target calibration, target distance measurement, robot moving, looking for the hole, fine-tuning the position, hitting the ball, etc.

\subsection{Calibrating the target}

After the camera, Bluetooth and upper computer connection is established successfully, the mouse is used for target calibration; the target is orange golf ball. After the success of Calibrating Target (as shown in Fig. 10), the box below will show all yellow feature points, as well as the calibration target image parameters. After successful calibration, there is only a small ball as feature point in robot's version. At this moment begins the operation of the overall strategy. The robot's position is shown on Fig. 11.

\subsection{Measuring the distance}

After calibrating target ball successfully, the robot can adjust head pitching Angle to $60^{\circ}$ to measure the distance. The location of the robot is not changed, just the camera Angle is changed in this process. The ball is in the vision central position and distance measurement is performed for the first time. 


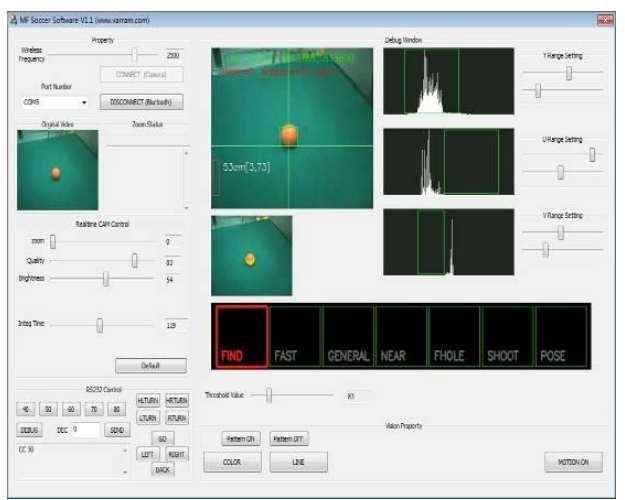

Fig. 10. Target's calibration

6.3. Moving close to the shot position

After the completion of the ranging, the program to the robot will have written policies according to the sent instructions. A close path is chosen according to the distance. When the distance is far, the robot chooses the way of striding forward quickly to shorten the distance to the ball.

\subsection{Looking for the hole}

Inevitably, deviation appear during the process of moving. This is the time to look for the hole and to locate the robot correctly, which ensures accurate hit of a golf ball to hole.

\subsection{Adjusting position and hitting the ball}

The final shooting process is again with the aim to the golf ball for fine tuning the robot's location, in order to make sure that the golf club can hit the golf ball. As shown on Fig. 13, the robot completes hitting the golf ball. Test results show that the system has reached the expected goal.

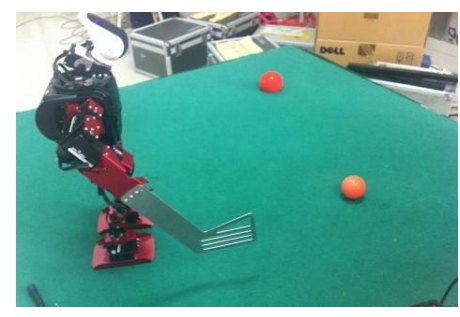

Fig. 11. Robot's initial position

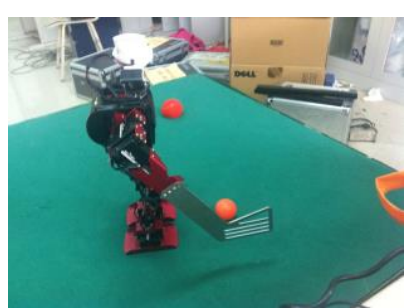

Fig. 12. looking for the hole

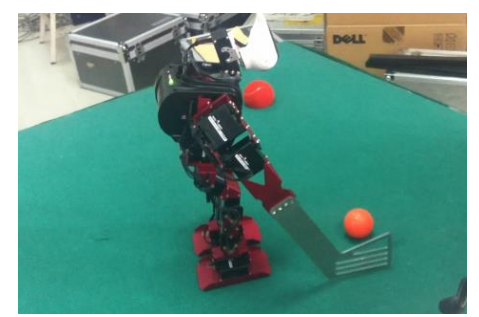

Fig. 13. Robot hiting the golf

\section{Conclusion}

Aiming at the golf tournament technical requirements in International Humanoid robot Olympic Game (IHOG), two-freedom-degrees "head \& eye" system based on monocular vision robot MF-AI is developed and described in this paper. The 
MF-AI robot only identifies the target in front of it, while the new system can identify the location of the golf ball and the hole, and can measure the distance between the two locations.

Experiments show that the two-freedom-degrees "head \& eye" system can identify targets, calibrate distance, measure robot moving, look for the hole, adjust the position and hit the golf ball. The system improves the accuracy of the golf ball hits into the hole effectively. In the 4th International Humanoid robot Olympic Game (IHOG 2013), the robot equipped with the "head \& eye" system got 11 out of 12 points and won the championship of the golf game.

\section{References}

1. Z h a n g, L.-G., S.-S. B i, J.-L. G a o. Human Motion Data Acquiring and Analyzing Method for Humanoid Robot Motion Designing. - Acta Automatica Sinica, Vol. 36, 2010, No 1, pp. 107-112.

2. T i m e s, A. Toyota Shows Range with Robot Violinist. - LA Times Business Pages, December 2007.

3. Gru n b e r g, D., R. E 11 e n b e rg, Y. E. K i m. From RoboNova to HUBO: Platforms for Robot Dance. - Progress in Robotics, 2009, Springer.

4. Ch e n, W., N. G a o, H. W a n g, D. S u n. Implementation of Tai Chi Chuan on Humanoid Robot. - IEEE/ICCSNT 2011, pp. 1128-1131.

5. Honda. Honda's Asmio Robot to Conduct the Detroit Symphony Orchestra. - Press Release, April 2008.

6. H o n g, B.-R., W.-D. K e. International Huo Olympic Games and Its Key Technology. - Journal of Harbin Institute of Technology, Vol. 17, 2010, pp. 1-4.

7. Ch e n, W., S. G a o, X. W u. HMCD-Based Dynamic Motion Strategy of Humanoid Robot on a Horizontal Bar. - CAAI Transactions on Intelligent Systems, Vol. 7, 2012, No 6, pp. 501505.

8. Z h o n g, Q.-B., B.-R. H o n g, Q.-S. P a n. Design and Implementation on Humanoid Robot Based on RoboBasic. - Journal of Harbin Institute of Technology, Vol. 41, 2009, pp. 28-32.

9. Li u, R. Z. OpenCV Tutorial, Beijing Aeronautics and Astronautics. University Press, Beijing, China, 2007.

10. $\mathrm{H}$ e, B. Visual $\mathrm{C}^{++}$Digital Image Processing. Beijing, China, People Post Press, 2001.

11. X u, C. M. Color Recognition Based on Machine Vision. - Mechanical Design and Manufacturing, Vol. 8, 2004, pp. 257-258. 